\title{
Integrity of lunar soil samples
}

To the Editor - We disagree with the conclusion by Cooper et al. ${ }^{1}$ that stored lunar soils from the Apollo missions have significantly degraded over time. Specifically, they directly compared particle size distributions of lunar soils obtained by two different techniques - a wet-sieving technique applied several decades ago versus a recent laser-diffraction technique applied to a selection of the same soils. They observed differences in the particle size distributions that led them to infer degradation of lunar soils over time.

However, we argue that a direct comparison of the lunar soil analyses obtained from these two measurement techniques is not valid.

Significant differences between the two techniques have been documented when applied to terrestrial soils ${ }^{2}$. Although Cooper et al. compared the techniques on a synthetic soil designed to be lunar-like ${ }^{3}$, this synthetic soil does not have the complexity of a space-weathered lunar soil or even a terrestrial-weathered soil on Earth ${ }^{4}$. We argue that the observed differences in particle size distributions result from differences between the two techniques, including the use of different carrier fluids (alcohol and water), as well as sample-size and sample-integrity biases. Thus, we claim that the Apollo era lunar soil samples have not degraded as reported.

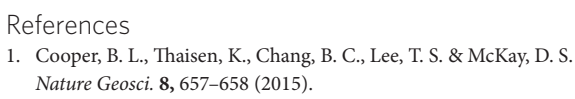

1. Cooper, B. L., Thaisen, K., Chang, B. C., Lee, T. S. \& McKay, D. S. Nature Geosci. 8, 657-658 (2015).

\author{
2. Eshel, G., Levy, G. J., Mingelgrin, U. \& Singer, M. J. \\ Soil Sci. Soc. Am. J. 68, 736-743 (2004). \\ 3. Stoeser, D. B., Rickman, D. L. \& Wilson, S. Design and \\ Specifications for the Highland Regolith Prototype Simulants \\ NU-LHT-1M and -2M (NASA, 2010). \\ 4. Taylor, L. A. \& Liu, Y. in Earth and Space 2010: Engineering, \\ Science, Construction, and Operations in Challenging Environments \\ (eds Song, G. \& Malla, R. B.) 106-118 (ASCE, 2010).
}

\author{
Lawrence A. Taylor ${ }^{1 \star}$, Yang Liu \\ and Gary Lofgren ${ }^{3}$ \\ 'Planetary Geosciences Institute, Earth \\ and Planetary Sciences, The University of \\ Tennessee, Knoxville, Tennessee 37996, \\ USA. ${ }^{2}$ Jet Propulsion Laboratory, California \\ Institute of Technology, Pasadena, California \\ 91109, USA. ${ }^{3}$ Johnson Space Center, Houston, \\ Texas 77058, USA. \\ *e-mail: lataylor@utk.edu
}

\section{Reply to 'Integrity of lunar soil samples'}

Cooper et al. reply - Taylor et al. argue that documented differences between laser diffraction and sieving techniques ${ }^{1}$, rather than degradation of the samples over time, can explain the different particle size distributions of lunar soil samples that we observe. However, we would expect comparatively larger particle sizes for laser diffraction measurements if this were the case; instead, our laser diffraction measurements yielded smaller values. We find that methodological differences cannot account for the discrepancy between the two sets of measurements.

Taylor et al. further suggest that a valid comparison between the measurement techniques on a lunar soil sample has not been demonstrated. Although not discussed in our paper ${ }^{2}$, we performed sieve and laser diffraction measurements for a representative material and found good agreement in the results. Taylor et al. argue that the material used lacks the complexity, mineralogy and texture of a natural terrestrial or lunar soil, however neither sieving nor laser diffraction techniques avoid uncertainties introduced by irregularly shaped particles ${ }^{1}$. Furthermore, mineralogy should not affect the laser diffraction measurements because minerals in the lunar soils do not interact with the carrier fluid (isopropanol). Various fluids were used in sieving, so the suggestion that water affected the sieve results applies to only a few cases.
References

1. Eshel, G., Levy, G. J., Mingelgrin, U. \& Singer, M. J. Soil Sci. Soc. Am. J. 68, 736-743 (2004).

2. Cooper, B. L., Thaisen, K., Chang, B. C., Lee, T. S. \& McKay, D. S Nature Geosci. 8, 657-658 (2015).

B. L. Cooper ${ }^{1 *}$, K. Thaisen², B. C. Chang', T. S. Lee ${ }^{1}$ and D. S. McKay ${ }^{3+}$ IInternational Space Exploration Research Institute, Hanyang University, 2nd Engineering Building, 1271, Sa 3-dong, Sangrok-gu, Ansan, Gyeonggi-do, 426-791, Korea. ${ }^{2}$ Geology Department, Grand Valley State University, Michigan 49401, USA. ${ }^{3}$ NASA Lyndon B. Johnson Space Center, Houston, Texas 77058, USA. 'Deceased.

*e-mail: bcooper108@gmail.com 